\title{
Avaliação das Habilidades Sociais de Crianças com Deficiência Intelectual sob a Perspectiva dos Professores ${ }^{1}$ \\ Social SKILLS EVALUATION OF CHILDREN WITH INTELLECTUAL DisABILITIES FROM THEIR TEACHERS’ PERSPECTIVE
}

\author{
Elizângela Fernandes FERREIRA² \\ Mey de Abreu van MUNSTER ${ }^{3}$
}

\begin{abstract}
RESUMO: o objetivo do presente estudo foi avaliar as habilidades sociais de alunos com deficiência intelectual, antes e após um programa de Educaçáo Física, sob a perspectiva dos professores. Desenvolveu-se sob abordagem quantitativa, com delineamento quase-experimental. A amostra foi composta por sete crianças com idade entre sete e 14 anos $(\mathrm{M}=10$; $\mathrm{DP}=2.61)$ e três professores com idade entre 24 e 45 anos $(\mathrm{M}=37.67$; DP=12.34). Os participantes foram avaliados pelos respectivos professores, por meio do instrumento Sistema de Avaliação das Habilidades Sociais (SSRS-BR), aplicado antes e após um programa de intervenção em Educação Física. O programa foi composto por 24 sessôes, com duração de 40 minutos cada, durante dois meses. As informações obtidas por meio do SSRS-BR foram analisadas quantitativamente pelo Método JT. Dos sete participantes, cinco obtiveram mudança positiva confiável nas habilidades sociais após o programa de Educação Física. A categoria social mais influenciada foi a responsabilidade, sendo que houve uma mudança positiva nas habilidades sociais globais. Conclui-se que após o programa de Educação Física as crianças com deficiência intelectual obtiveram um aumento no escore global das habilidades sociais.
\end{abstract}

PALAVRAS-CHAVE: Educação Especial. Educação Física. Habilidades Sociais.

\begin{abstract}
The aim of this study was to evaluate the social skills of students with intellectual disabilities, before and after a Physical Education program, from the teachers' perspective. Under a quantitative approach, a research with quasi-experimental design was developed. The sample consisted of seven children aged between seven and 14 years $(M=10, S D=2.61)$ and three teachers aged between 24 and 45 years $(M=37.67, S D=12.34)$. Participants were evaluated by these teachers through the instrument System Evaluation of Social Skills (SSRS-BR), applied before and after an intervention program in Physical Education. The program consisted of 24 sessions lasting 40 minutes each, during two months. Information obtained through the SSRS- BR was analyzed quantitatively by JT method. Five out of the seven participants obtained reliable positive change in social skills after the physical education program. The most influenced social class was responsibility, and there was a positive change in overall social skills. It was concluded that after attending the Physical Education program, children with intellectual disabilities obtained an increase in the overall score of social skills.
\end{abstract}

KEYWORDS: Special Education. Physical Education. Social Skills.

\section{INTRODUÇÃo}

As habilidades sociais (HS) não apresentam uma definição consensual entres autores (BOLSONI-SILVA, 2002; SMITH; MATSON, 2010; COOK; OLIVER, 2011). Numerosas definições acerca das habilidades sociais foram construídas ao longo dos últimos trinta anos (DEL PRETTE; DEL PRETTE, 2009). Contudo, Del Prette e Del Prette (2011, p.31) conceituam as HS como "diferentes classes de comportamentos sociais do repertório de um indivíduo, que contribuem para a competência social, favorecendo um relacionamento saudável e produtivo com as demais pessoas".

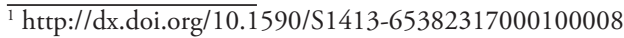

${ }^{2}$ Docente da Faculdade Governador Ozanam Coelho. Santo Antônio, Viçosa, MG, Brasil. elizangel.fernandes.f@gmail.com

${ }^{3}$ Docente do Programa de Pós-Graduação em Educação Especial da Universidade Federal de São Carlos, São Carlos, SP, Brasil. munster.mey@gmail.br
} 
Atualmente, há uma preocupação com as HS na infância devido a diversidade de estímulos e cobranças que cercam as crianças (DEL PRETTE; DEL PRETTE, 2011). Pesquisas têm demonstrado que crianças apresentam déficits nas habilidades sociais e uma alta frequência de problemas de comportamento (DEL PRETTE; BARRETO; FREITAS, 2011). Entretanto, programas de treinamento das HS são eficazes para desenvolvimento de comportamentos sociais desejáveis e redução dos problemas de comportamento (GONÇALVES; MURTA, 2008).

As pessoas que não conseguem desenvolver as HS adequadamente apresentam maiores riscos de obter resultados negativos no decorrer da vida, incluindo a rejeição de pares, manifestaçóes de transtornos psicológicos, abandono da escola, criminalidade e baixo rendimento escolar (GRESHAM; VAN; COOK, 2006; DEL PRETTE; DEL PRETTE, 2011). As relaçóes sociais positivas influenciam o desenvolvimento intelectual, comunicativo, interpessoal e emocional das pessoas (FREITAS; DEL PRETTE, 2010a; DE FREITAS, 2012; VAN NIEUWENHUIJZEN; VRIENS, 2012). Assim, trabalhar a aquisição e o aprimoramento das HS é fundamental para todas as pessoas, incluindo aquelas com deficiência intelectual.

As crianças com deficiência intelectual podem apresentar comprometimentos cognitivos, afetivos, perceptivos ou motores que dificultam o desenvolvimento das HS (DEL PRETTE; DEL PRETTE, 2011). Já na primeira infância, as crianças com deficiência intelectual podem evidenciar sinais de distúrbios no desenvolvimento sensório-motor, da fala e cognição, os quais ocasionam inadequaçóes nos contatos sociais (JAPUNDZA-MILISAVLJEVIC; DJURIC-ZDRAVKOVIC; MACESIC-PETROVIC, 2010).

Pesquisadores reconhecem a relevância do desenvolvimento das HS para essa população enfatizando a melhora na qualidade de vida, bem-estar e a capacidade de participar na comunidade (PARK; LOMAN; MILLER, 2008; COOK; OLIVER, 2011; DEL PRETTE; DEL PRETTE, 2011). As crianças com deficiência intelectual apresentam respostas emocionais e relações interpessoais mais pobres em relação às crianças sem deficiência (SANTOS, 2007), além de evitar o contato social e visual, demonstrar dificuldade em seguir instruçóes simples e interromper as atividades dos colegas (MATSON et al., 2003; SMITH; MATSON, 2010). As crianças com deficiência intelectual apresentam níveis ainda menores de interação social quando comparadas às crianças da sua idade na população geral (JAPUNDZA-MILISAVLJEVIC; DJURIC-ZDRAVKOVIC; MACESIC-PETROVIC, 2010).

Assim, justifica-se a importância de se desenvolver programas com a finalidade de promover um repertório diversificado de HS junto a essa população (DEL PRETTE; DEL PRETTE, 2011). A promoção de HS auxilia na interação social, competência social e enfrentamento da baixa autoestima e da rejeição social (PARK; LOMAN; MILLER, 2008).

Diante dos obstáculos no desenvolvimento das HS, mais especificamente nas interaçóes sociais estabelecidas por pessoas com deficiência intelectual, destaca-se a Educação Física como um possível catalisador para promovê-las. A Educação Física, enquanto componente curricular do ensino básico consiste em uma disciplina desenvolvida principalmente em um contexto de grupo, pois a maioria das atividades são desenvolvidas coletivamente, favorecendo a interação com o outro, estimulando as interações sociais, o contato visual entre alunos e professor, além de possibilitar o reforço das habilidades acadêmicas, assim como outras classes das HS. 
Nesse sentido, a Educação Física proporciona benefícios motores, sociais e fisiológicos durante a sua prática (CIDADE; FREITAS, 2002; CASTELLANI FILHO et al., 2009). Mauerberg-de-Castro (2011) menciona a importância de se estimular a independência nas brincadeiras, incentivar a cooperação e receptividade às convençóes sociais nas pessoas com deficiência intelectual, durante as intervençóes próprias da Educação Física. Além das brincadeiras, a Educação Física dispóe de conteúdos como o Jogo, a Ginástica, a Dança, a Luta e o Esporte (CASTELLANI FILHO et al., 2009), os quais possuem características que propiciam o desenvolvimento das HS.

No âmbito das HS, há pesquisas com o enfoque em avaliar as HS das pessoas com Deficiência Intelectual (DI) (BARBOSA; DEL PRETTE, 2002; PÉREZ-RAMOS et al., 2003; ROSIN-PINOLA; DEL PRETTE; DEL PRETTE, 2007; FREITAS; DEL PRETTE, 2010b; COOK; OLIVER, 2011), como também, o nível de habilidades sociais entre pais cujos filhos possuem DI (CARDOZO; SOARES, 2010). No entanto há poucas pesquisas envolvendo a interface Educação Física e Habilidades Sociais; estas tornam-se ainda mais escassas entre a população com Deficiência Intelectual. Os estudos envolvendo EF e DI têm sido direcionados à área de comportamento motor (GORLA; RODRIGUES; PEREIRA, 2002; BIANCONI; MUNSTER, 2011; MAUERBERG-DE CASTRO et al., 2013).

Diante do exposto, o objetivo deste estudo foi avaliar as habilidades sociais de alunos com deficiência intelectual, antes e após um programa de Educação Física, sob a perspectiva dos professores.

\section{Método}

Trata-se de pesquisa com delineamento quase-experimental pois, embora não envolva distribuiçãao aleatória dos sujeitos e tampouco se utilize de grupos controle, prevê a "comparação entre as condiçóes de tratamento e não tratamento" com os mesmos sujeitos antes e após o tratamento, permitindo alguma compreensão acerca de como as variáveis se relacionam (GIL, 2008, p.54). Nesse caso, pretendeu-se avaliar as habilidades sociais (variável dependente) em um mesmo grupo de crianças com deficiência intelectual, comparando-se os dados anteriores e posteriores à intervenção baseada em um programa de Educação Física (variável independente).

\subsection{Participantes}

Participaram do estudo um grupo de sete crianças (cinco meninas e dois meninos), com idade entre sete e 14 anos $(\mathrm{M}=10$, DP $=2.61)$, matriculadas em uma escola especial localizada no centro oeste paulista do Brasil. Para inclusão na amostra, o critério foi apresentar deficiência intelectual ${ }^{4}$, sendo o diagnóstico obtido por meio dos prontuários disponíveis na instituição de ensino onde foi desenvolvida a pesquisa. Além das crianças com deficiência intelectual, participaram do estudo três professores contratados pela referida instituição, os quais costumavam ministrar aulas para as crianças envolvidas no estudo. Tais profissionais atuavam

\footnotetext{
${ }^{4}$ Incapacidade caracterizada por limitaçôes significativas tanto no funcionamento intelectual quanto no comportamento adaptativo expresso em habilidades conceituais, sociais e práticas, sendo esta incapacidade com origem antes da idade dos 18 anos (SCHALOCK et al., 2010).
} 
como professores de sala $(n=1)$, educação física $(n=1)$ e expressão corporal $(n=1)$, apresentando faixa etária entre 24 e 45 anos $(\mathrm{M}=37.67$; $\mathrm{DP}=12.34)$.

O estudo foi conduzido de acordo com as exigências da Resolução no466/12 do Conselho Nacional de Saúde, tendo sido submetido e aprovado pelo Comitê de Ética em Pesquisa com Seres Humanos da Universidade Federal de São Carlos (Parecer No 03989312.4.0000.5504).

\subsection{InSTRUMENTOS DE COLETA DE DADOS}

Cada um dos participantes foi avaliado por meio do Sistema de Avaliação de Habilidades Sociais - SSRS, criado por Gresham e Elliott em 1990, porém usado em versão adaptada para o Brasil (SSRS-BR) (BANDEIRA et al., 2005).

O SSRS-BR é constituído por um conjunto de formulários que permite avaliar habilidades sociais, problemas de comportamento e desempenho acadêmico. Pode ser aplicado em alunos do ensino infantil e do ensino fundamental. O emprego do SSRS-BR neste estudo justifica-se pelos índices normativos validados para a população com Deficiência Intelectual (FREITAS; DEL PRETTE, 2010b).

Os formulários do SSRS-BR são apresentados em três versões: para autoavaliação do aluno, para avaliação por pais e por professores. Neste estudo foi utilizado somente o formulário de preenchimento por professores. Espera-se que o professor avalie cada aluno quanto à frequência de emissão de habilidades sociais (30 itens) Alfa Conbach $=0,94$, frequência de comportamentos problemáticos (18 itens) e frequência de competência acadêmica (nove itens), bem como a importância dos itens de habilidades sociais. A escala de frequência é respondida com base em três pontos (nunca = zero; algumas vezes $=$ um e muito frequentemente $=$ dois) para a emissão das HS, e para a escala de importância atribui-se a pontuação (não importante, importante e indispensável). Já na escala de competência acadêmica, o professor deve comparar o aluno com as demais crianças de sua sala, obedecendo a um critério e escolhendo um dos seguintes pontos (entre os $10 \%$ piores, $20 \%$ piores, $40 \%$ médios, $20 \%$ bons ou entre os $10 \%$ ótimos) para os itens, leitura, matemática, motivação geral, participação dos pais, funcionamento intelectual e comportamento geral em classe. Entretanto, ressalta-se que como o presente estudo focou as HS, não foram avaliados os itens referentes à competência acadêmica e ao comportamento problemático.

\subsection{Procedimentos}

A intervenção foi composta por 24 sessóes com duração de quarenta minutos, desenvolvidas ao longo de dois meses (frequência = três vezes na semana). Todos os sete integrantes do grupo frequentaram o programa de Educação Física proposto como intervenção pela presente pesquisa, com assiduidade satisfatória.

Após os trâmites éticos, os participantes selecionados para o estudo foram envolvidos em três momentos: a avaliação inicial, a intervenção e a avaliação final, sendo as avaliações inicial e final, realizadas por meio do SSRS-BR. O formulário foi preenchido pelos professores 
de sala, de Educação Física e de Expressão Corporal, antes e após o período de intervenção correspondente ao desenvolvimento do programa de Educação Física.

As medidas obtidas pelo SSRS-BR foram realizadas antes de iniciar a primeira sessão do programa e após a última sessão $(\mathrm{N}=24)$.

\subsection{Programa de Educaçáo Física}

Após a apreciação e aprovação do programa de pesquisa pela coordenação da instituição, e realização da avaliação inicial pelos professores, teve início a fase de intervenção, caracterizada pela aplicação do Programa de Educação Física. As sessões de intervenção foram realizadas pela primeira pesquisadora, a qual possui formação inicial em Educação Física.

O Programa de Educação Física teve como finalidade estimular a aquisição e aprimoramento de habilidades sociais em crianças com deficiência intelectual matriculadas no ensino especial, por meio de conteúdos próprios da Educação Física.

O conteúdo programático da intervenção foi diversificado, envolvendo elementos da cultura corporal de movimento, em associação a aspectos relacionados às habilidades sociais. O planejamento das sessóes foi desenvolvido com participação institucional, procurando considerar o calendário de eventos e comemorações cívicas. A distribuição do conteúdo ao longo das sessóes de intervenção pode encontra-se descrito no Quadro 1.

\begin{tabular}{|c|c|}
\hline Conteúdo & Descrição \\
\hline $\begin{array}{l}\text { Dança } \\
\text { (Sete sessóes) }\end{array}$ & $\begin{array}{l}\text { Tema: Dança indígena } \\
\text { As atividades de dança permitiram trabalhar ritmo, as açôes da vida diária, estados afe- } \\
\text { tivos e sensaçóes corporais, sendo associadas ao significado do trabalho, saúde, costumes } \\
\text { sociais dos povos indígenas. A Dança Indígena foi abordada na perspectiva da dança } \\
\text { educativa, a qual advoga que a funçáo da dança no ambiente escolar é formar pessoas } \\
\text { livres e capazes de expressar atitudes criativas e conscientes o fluxo natural do movimen- } \\
\text { to humano (LABAN, 1990). }\end{array}$ \\
\hline $\begin{array}{l}\text { Jogos } \\
\text { (Cinco sessôes) }\end{array}$ & $\begin{array}{l}\text { Tema: Jogos cooperativos } \\
\text { O conteúdo Jogo foi baseado em aspectos como a ludicidade, a cooperação e os signifi- } \\
\text { cados dos jogos cooperativos para o aluno, sendo abordado por constituir um elemento } \\
\text { facilitador para o desenvolvimento das classes das HS pretendidas. De acordo com } \\
\text { Andrade e Holsbach (2001) os jogos e as brincadeiras tornam possível que a criança } \\
\text { desenvolva a inteligência, atençáo, concentraçáo, linguagem, sociabilidade, percepçóes e } \\
\text { habilidades em geral. Nesse sentido, este conteúdo enfatizou os aspectos do conhecimen- } \\
\text { to de si mesmo, de estabelecer as relaçóes sociais, favorecer açóes de cooperação, além } \\
\text { das relaçóes espaço-temporais, por meio dos jogos cooperativos. }\end{array}$ \\
\hline $\begin{array}{l}\text { Ginástica Geral } \\
\text { (Quatro sessōes) }\end{array}$ & $\begin{array}{l}\text { Tema: Circo } \\
\text { Os elementos relacionados ao circo podem ser considerados como fundamentos básicos } \\
\text { da ginástica: o "saltar", "equilibrar", "rolar/girar", "trepar" e "balançar/embalar". A esco- } \\
\text { lha por esses elementos justifica-se por serem atividades com significado histórico-social, } \\
\text { além de contribuir com o desenvolvimento motor das crianças com DI. Os fundamen- } \\
\text { tos da ginástica geral quando adequadamente trabalhados permitem desenvolver capaci- } \\
\text { dades físicas e motoras, como o aspecto sócio afetivo, além de favorecer a expressividade } \\
\text { emocional durante a sua prática. }\end{array}$ \\
\hline
\end{tabular}




\begin{tabular}{|l|l|}
\hline $\begin{array}{l}\text { Esporte } \\
\text { (Seis sessões) }\end{array}$ & $\begin{array}{l}\text { Tema: jogos pré-desportivos } \\
\text { Geralmente empregados no início do processo de adaptação aos esportes, os jogos } \\
\text { pré-desportivos envolvem a participação coletiva e o respeito às convençóes (regras) } \\
\text { estabelecidas pelo grupo. Em relaçào às HS, a finalidade foi favorecer um ambiente } \\
\text { onde os alunos pudessem vivenciar sentimentos de derrota, vitória em grupo, cooperar } \\
\text { com sua equipe, criação e respeito às regras, além de expressar seus sentimentos durante } \\
\text { os momentos de aula e avaliação. }\end{array}$ \\
\hline $\begin{array}{l}\text { Luta } \\
\text { (Duas sessões) }\end{array}$ & $\begin{array}{l}\text { A Luta, não foi abordada em seu caráter esportivo, mas sim enfatizando os aspectos } \\
\text { socioeducativos. As principais características trabalhadas nesse tema foram os princípios } \\
\text { de valores das lutas, modos de comportamento, autonomia, relaçóes coletivas, saudação/ } \\
\text { civilidade e enfretamento. }\end{array}$ \\
\hline
\end{tabular}

Quadro 1 - Distribuição do conteúdo no decorrer da intervenção.

Fonte: elaboração própria.

As situações de ensino envolveram diversas estratégias de ensino e vários materiais lúdicos:

Em síntese, as estratégias de ensino empregadas foram:

- Posicionamento do professor durante a explicação da atividade, geralmente era em formato de círculo (roda), com o intuito de estabelecer o contato visual com todos os alunos;

- Instruçóes claras e explicaçóes objetivas das tarefas;

- Diminuição do tempo de duração das atividades, visando aumentar a motivação e envolvimento dos alunos com a tarefa;

- Modulação da voz de comando, de forma a destacar as situaçóes que mereciam mais atenção durante a realização da atividade: em alguns momentos era preciso prender a atenção do aluno, por meio da diferenciação na entonação de voz;

- Permissão para que o aluno se expressasse durante a transição de uma atividade para outra, até mesmo, com sugestóes de novas formas de realizar a atividade anteriormente proposta;

- Retomada do conteúdo anterior, relembrando as atividades das aulas passadas, no início da intervenção, com o intuito de relacionar o conhecimento apreendido com o que seria proposto.

Como recursos materiais, foram utilizados: petecas e bolas de papéis em cores vivas, confeccionadas pelos próprios alunos; bolas de plástico de dimensóes variadas; bola de basquete; materiais circences (bolas para malabares, arco, fitas, chapéus, perucas e nariz de palhaço); balóes de látex, bambolês, jornal e aparelho de som com músicas variadas.

Cada sessão apresentava uma estrutura padronizada e subdividida em três momentos, conforme apresentado no Quadro 2: 


\begin{tabular}{|l|l|}
\hline Apresentação do tema & $\begin{array}{l}\text { Na parte inicial, ou apresentação, cabia informar aos participantes o que seria } \\
\text { trabalhado naquela sessão, além de identificar o conhecimento prévio do aluno } \\
\text { sobre o tema. }\end{array}$ \\
\hline Desenvolvimento & $\begin{array}{l}\text { O desenvolvimento era composto pela vivência das atividades corporais individuais, } \\
\text { cooperativas (duplas, trios e grupo) e de enfrentamento, as quais foram abordadas } \\
\text { por meio dos conteúdos jogo, luta, ginástica, esporte e dança, criaçáo de diferentes } \\
\text { recursos pedagógicos (por exemplo, bolas, petecas, bexigas, cordas etc.). }\end{array}$ \\
\hline Avaliação & $\begin{array}{l}\text { A fase de avaliação era caracterizada pela discussão e reflexão sobre a participação } \\
\text { e interação obtida naquela sessão. }\end{array}$ \\
\hline
\end{tabular}

Quadro 2 - Estrutura das sessões de intervenção.

Fonte: elaboração própria.

Maiores informações acerca do Programa de Educação Física e o detalhamento de cada sessão (objetivo, conteúdo, estratégias e materiais) encontram-se descritos em Ferreira (2014).

\subsection{ANÁlise Estatística}

Para análise dos dados quantitativos obtidos pelo SSRS-BR foi empregado o Método JT, proposto por Jacobson e Truax (1991). Esse método estatístico tem sido empregado em pesquisas e intervençóes com sujeitos únicos ou em amostras reduzidas (DE AGUIAR et al., 2010; DE SÁ; DEL PRETTE, 2012; FERREIRA; DEL PRETTE, 2013). Segundo Villa, Aguiar e Del Prette (2012), o Método JT é voltado para as pesquisas que visam investigar a confiabilidade das mudanças pré e pós-intervenção e a significância clínica dessas mudanças, além de representar uma alternativa para as pesquisas/intervençóes com grupos pequenos e não possuir um delineamento com grupo controle.

A análise realizada por meio do Método JT esteve apoiada em seus dois conceitos centrais: o Índice de Mudança Confiável (IMC) e a Significância Clínica (SC), os quais possuem equaçóes específicas para o seu cálculo (JACOBSON; TRUAX, 1991; DEL PRETTE; DEL PRETTE, 2008; VILLA; AGUIAR; DEL PRETTE, 2012). Para os cálculos do Método JT, foi considerado o escore global, juntamente com as classes das habilidades sociais, tendo sido utilizadas a média e o desvio padrão com base na amostra local (sete participantes).

Para o cálculo do ponto de corte de significância clínica utilizou-se o critério A, que, segundo Jacobson e Truax (1991), é empregado quando não se dispóe de dados normativos da população funcional. Para a compreensão dos resultados quanto à confiabilidade das mudanças, deve-se observar a linha diagonal central que separa acima as diferenças positivas (pós > pré) e abaixo as negativas (pós < pré). Há ainda duas diagonais tracejadas que delimitam uma área de incerteza, na qual não é possível afirmar que houve mudança confiável. Quanto à significância clínica, a análise dos gráficos é realizada por meio de quatro quadrantes formados pelo cruzamento da linha vertical com a horizontal. Para afirmar que houve uma mudança clinicamente significativa (na qual a população clínica passou a ser não clínica), os participantes devem estar localizados no quadrante acima das linhas horizontais e à esquerda das linhas verticais. 
Com o intuito de verificar se as HS apresentaram um efeito positivo após o programa de Educação Física, foram calculadas as médias pré e pós-intervenção realizadas por meio do SSRS-BR, obtidas pelas avaliaçóes realizadas pelos professores de Sala, de Expressão Corporal e Educação Física.

\section{Resultados}

A Tabela 1 demonstra as médias das avaliações pré e pós-intervenção de cada um dos participantes, obtidas pelo SSRS BR. As médias pré e pós-intervenção são resultantes dos índices atribuídos pelos Professores de Sala (PS), de Educação Física (EF) e de Expressão Corporal (EC).

Tabela 1 - Resultados obtidos pelo SSRS BR correspondentes às habilidades sociais individuais.

\begin{tabular}{|c|c|c|c|c|c|c|c|c|c|}
\hline \multirow{3}{*}{$\mathbf{N}$} & \multirow{3}{*}{$\begin{array}{l}\text { HABILIDADES } \\
\text { SOCIAIS }\end{array}$} & \multicolumn{6}{|c|}{ PROFESSORES } & \multirow{2}{*}{\multicolumn{2}{|c|}{ MÉDIA }} \\
\hline & & \multicolumn{2}{|l|}{ PS } & \multicolumn{2}{|l|}{ EF } & \multicolumn{2}{|l|}{ EC } & & \\
\hline & & PRE & POS & PRE & POS & PRE & POS & PRE & POS \\
\hline \multirow[t]{2}{*}{ P1 } & $\begin{array}{l}\text { Responsabilidade } \\
\text { AsserçãoPositiva } \\
\text { Autocontrole } \\
\text { Autodefesa } \\
\text { Cooperação }\end{array}$ & $\begin{array}{l}20 \\
14 \\
8 \\
1 \\
6\end{array}$ & $\begin{array}{l}23 \\
10 \\
12 \\
1 \\
4\end{array}$ & $\begin{array}{l}22 \\
8 \\
11 \\
1 \\
4\end{array}$ & $\begin{array}{l}24 \\
9 \\
12 \\
1 \\
4\end{array}$ & $\begin{array}{l}18 \\
9 \\
15 \\
1 \\
3\end{array}$ & $\begin{array}{l}20 \\
8 \\
16 \\
3 \\
3\end{array}$ & $\begin{array}{l}20 \\
10,33 \\
11,33 \\
1 \\
4,33\end{array}$ & $\begin{array}{l}22,33 \\
9 \\
13,33 \\
1,66 \\
3,66\end{array}$ \\
\hline & Global & 35 & 38 & 35 & 38 & 32 & 38 & 34 & 38 \\
\hline \multirow[t]{2}{*}{ P2 } & $\begin{array}{l}\text { Responsabilidade } \\
\text { AsserçáoPositiva } \\
\text { Autocontrole } \\
\text { Autodefesa } \\
\text { Cooperação }\end{array}$ & $\begin{array}{l}14 \\
12 \\
5 \\
2 \\
4\end{array}$ & $\begin{array}{l}16 \\
9 \\
10 \\
1 \\
3\end{array}$ & $\begin{array}{l}14 \\
12 \\
5 \\
2 \\
4\end{array}$ & $\begin{array}{l}14 \\
6 \\
9 \\
1 \\
1\end{array}$ & $\begin{array}{l}14 \\
12 \\
5 \\
2 \\
4\end{array}$ & $\begin{array}{l}14 \\
3 \\
11 \\
1 \\
1\end{array}$ & $\begin{array}{l}14 \\
12 \\
5 \\
3 \\
4\end{array}$ & $\begin{array}{l}14,66 \\
6 \\
10 \\
1,66\end{array}$ \\
\hline & Global & 28 & 30 & 28 & 25 & 28 & 23 & 28 & 26 \\
\hline \multirow[t]{2}{*}{ P3 } & $\begin{array}{l}\text { Responsabilidade } \\
\text { AsserçãoPositiva } \\
\text { Autocontrole } \\
\text { Autodefesa } \\
\text { Cooperação }\end{array}$ & $\begin{array}{l}18 \\
13 \\
7 \\
2 \\
4\end{array}$ & $\begin{array}{l}20 \\
13 \\
5 \\
2 \\
7\end{array}$ & $\begin{array}{l}16 \\
10 \\
5 \\
3 \\
2\end{array}$ & $\begin{array}{l}23 \\
11 \\
10 \\
2 \\
5\end{array}$ & $\begin{array}{l}6 \\
7 \\
3 \\
1 \\
2\end{array}$ & $\begin{array}{l}20 \\
15 \\
11 \\
4 \\
6\end{array}$ & $\begin{array}{l}13,33 \\
10 \\
5 \\
2 \\
2,66\end{array}$ & $\begin{array}{l}21^{*} \\
13 \\
8,66 \\
2,66 \\
6\end{array}$ \\
\hline & Global & 34 & 34 & 31 & 38 & 13 & 41 & 26 & $37,66^{*}$ \\
\hline \multirow[t]{2}{*}{ P4 } & $\begin{array}{l}\text { Responsabilidade } \\
\text { AsserçãoPositiva } \\
\text { Autocontrole } \\
\text { Autodefesa } \\
\text { Cooperaçâo }\end{array}$ & $\begin{array}{l}17 \\
8 \\
4 \\
0 \\
6\end{array}$ & $\begin{array}{l}17 \\
9 \\
9 \\
1 \\
5\end{array}$ & $\begin{array}{l}16 \\
2 \\
5 \\
0 \\
2\end{array}$ & $\begin{array}{l}21 \\
6 \\
11 \\
1 \\
3\end{array}$ & $\begin{array}{l}12 \\
6 \\
10 \\
1 \\
2\end{array}$ & $\begin{array}{l}18 \\
5 \\
11 \\
0 \\
4\end{array}$ & $\begin{array}{l}15 \\
5,33 \\
6,33 \\
0 \\
3,33\end{array}$ & $\begin{array}{l}18,66 \\
6,66 \\
10,33 \\
1 \\
4\end{array}$ \\
\hline & Global & 25 & 30 & 20 & 32 & 22 & 29 & 22,33 & $30,33^{*}$ \\
\hline \multirow[t]{2}{*}{ P5 } & $\begin{array}{l}\text { Responsabilidade } \\
\text { AsserçãoPositiva } \\
\text { Autocontrole } \\
\text { Autodefesa } \\
\text { Cooperação }\end{array}$ & $\begin{array}{l}20 \\
8 \\
10 \\
1 \\
3\end{array}$ & $\begin{array}{l}24 \\
13 \\
11 \\
2 \\
6\end{array}$ & $\begin{array}{l}18 \\
10 \\
11 \\
1 \\
4\end{array}$ & $\begin{array}{l}28 \\
14 \\
15 \\
2 \\
7\end{array}$ & $\begin{array}{l}16 \\
5 \\
15 \\
2 \\
2\end{array}$ & $\begin{array}{l}26 \\
10 \\
17 \\
2 \\
6\end{array}$ & $\begin{array}{l}18 \\
7,66 \\
12 \\
1,33 \\
3\end{array}$ & $\begin{array}{l}26^{*} \\
12,33 \\
14,33 \\
2 \\
6,33\end{array}$ \\
\hline & Global & 32 & 41 & 33 & 48 & 28 & 45 & 31 & $44,66^{*}$ \\
\hline \multirow[t]{2}{*}{ P6 } & $\begin{array}{l}\text { Responsabilidade } \\
\text { AsserçãoPositiva } \\
\text { Autocontrole } \\
\text { Autodefesa } \\
\text { Cooperação }\end{array}$ & $\begin{array}{l}27 \\
14 \\
15 \\
1 \\
7\end{array}$ & $\begin{array}{l}28 \\
16 \\
16 \\
4 \\
7\end{array}$ & $\begin{array}{l}26 \\
11 \\
12 \\
1 \\
7\end{array}$ & $\begin{array}{l}29 \\
14 \\
16 \\
2 \\
8\end{array}$ & $\begin{array}{l}19 \\
10 \\
16 \\
3 \\
3\end{array}$ & $\begin{array}{l}28 \\
13 \\
18 \\
4 \\
8\end{array}$ & $\begin{array}{l}24 \\
11,66 \\
14,33 \\
1,66 \\
5,66\end{array}$ & $\begin{array}{l}28,33 \\
14,33 \\
16,66 \\
3,33 \\
7,66\end{array}$ \\
\hline & Global & 48 & 53 & 41 & 51 & 38 & 53 & 42,33 & $52,33^{*}$ \\
\hline \multirow[t]{2}{*}{ P7 } & $\begin{array}{l}\text { Responsabilidade } \\
\text { AsserçãoPositiva } \\
\text { Autoçontrole } \\
\text { Autodefesa } \\
\text { Cooperaçẫo }\end{array}$ & $\begin{array}{l}22 \\
16 \\
12 \\
3 \\
7\end{array}$ & $\begin{array}{l}24 \\
17 \\
11 \\
5 \\
6\end{array}$ & $\begin{array}{l}22 \\
4 \\
13 \\
1 \\
2\end{array}$ & $\begin{array}{l}25 \\
8 \\
13 \\
2 \\
3\end{array}$ & $\begin{array}{l}15 \\
5 \\
14 \\
1 \\
2\end{array}$ & $\begin{array}{l}27 \\
14 \\
18 \\
3 \\
7\end{array}$ & $\begin{array}{l}19,66 \\
8,33 \\
13 \\
1,66 \\
3,66\end{array}$ & $\begin{array}{l}25,33 \\
13 \\
14 \\
3,33 \\
5,33\end{array}$ \\
\hline & Global & 44 & 46 & 33 & 40 & 25 & 52 & 34 & $46^{*}$ \\
\hline
\end{tabular}

Legenda: *Mudança positiva confiável.

Fonte: elaboração própria.

Destaca-se que, entre os sete participantes, dois (P3 e P5) obtiveram mudança positiva confiável na classe Responsabilidade. Quanto às habilidades sociais globais, obtida pela 
média entre as cinco HS específicas, cinco participantes (P3, P4, P5, P6 e P7) apresentaram resultados significantes.

A Figura 1 apresenta as Habilidades Sociais Globais pós-intervenção de cada um dos participantes, de acordo com a avaliação dos professores. Verifica-se que, segundo a média atribuída pelos professores, cinco participantes (P3, P4, P5, P6, P7) obtiveram uma mudança positiva confiável (MPC) após o programa, podendo-se inferir que houve melhora estatisticamente significante nas Habilidades Sociais Globais dos mesmos. Dois dos participantes (P1 e P2) permaneceram na zona de ausência de mudança (AM).

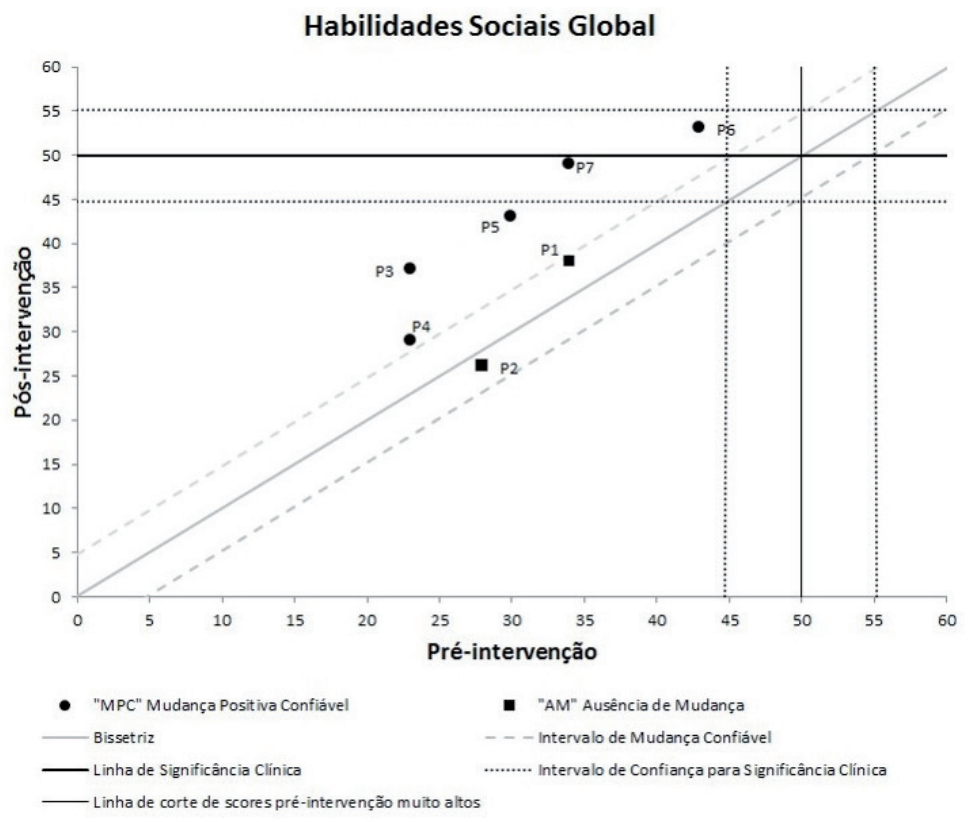

Figura 1 - Gráfico referente à avaliação das Habilidades Sociais Globais dos participantes após intervenção.

Fonte: elaboração própria.

Ainda na Figura 1 observa-se que os participantes P5, P6 e P7 atingiram o intervalo de Significância Clínica, com destaque para P6, que ainda na avaliação pré-intervenção obteve uma pontuação alta (41) nas Habilidades Sociais Globais.

O gráfico representado na Figura 2 demonstra que todas as classes das Habilidades Sociais obtiveram um aumento após o período de intervenção correspondente ao programa de Educação Física; entretanto, não houve mudança positiva confiável. Pode-se verificar que a classe Autodefesa (77\%) foi a que sofreu maior influência, enquanto a Asserção Positiva (16\%) foi a menos influenciada. As demais habilidades sociais específicas registraram as seguintes mudanças: Autocontrole (26\%); Cooperação (26\%); e Responsabilidade (24\%). 


\section{Categorias das Habilidades Sociais}

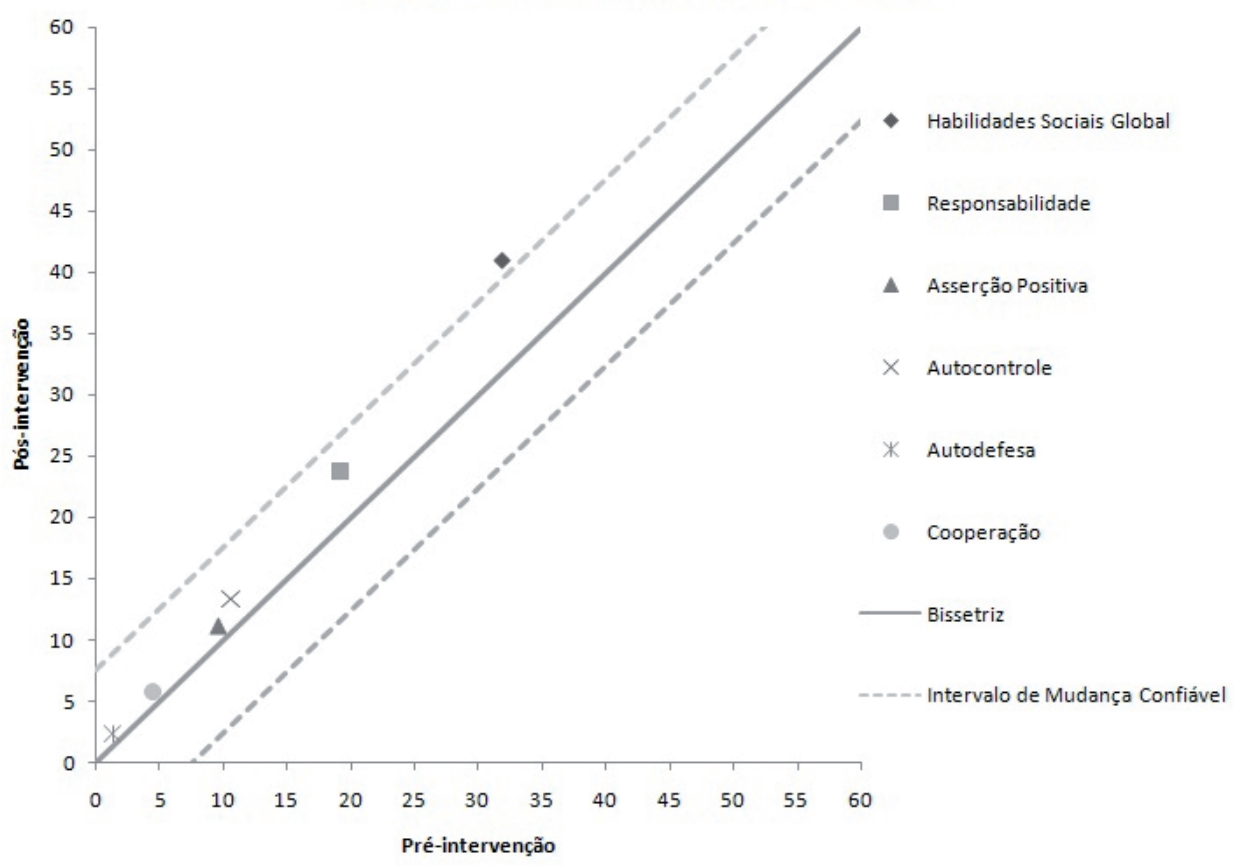

Figura 2 - Gráfico correspondente ao desempenho dos participantes nas classes específicas das Habilidades Sociais.

Fonte: elaboração própria.

Os dados obtidos pelo SSRS BR revelam que o programa de Educação Física influenciou aquisição e o aprimoramento das Habilidades Sociais da maioria dos participantes e incidiu de forma estatisticamente significante no escore Global das Habilidades Sociais.

\section{Discussão}

Os efeitos decorrentes de intervenção em Educação Física junto a pessoas com deficiência intelectual têm demonstrado resultados positivos, sobretudo quanto ao desempenho motor (GORLA; RODRIGUES; PEREIRA, 2002). Embora na literatura não tenham sido identificados estudos precedentes abordando a temática "Educação Física e Habilidades Sociais", Mauerberg-de-Castro et al. (2013) ressalta que os programas de Educação Física podem contribuir para o aprimoramento de condutas sociais por pessoas com deficiências intelectuais.

A partir dos dados obtidos por meio do SSRS-BR (avaliaçáo realizada pelos professores), verificou-se evolução nas HS de crianças com DI após o programa de Educação Física. Os resultados deste estudo indicam que as atividades de Educação Física podem ser eficazes para aquisição e aprimoramento das habilidades sociais de crianças com DI. Os dados encontrados corroboram com a noçáo de que a Educaçáo Física apresenta efeitos positivos sobre as habilidades sociais em pessoas com DI, como os relatados na população em geral e em grupos 
clinicamente definidos (BLUECHARDT; SHEPHARD, 1995; GOUDAS; MAGOTSIOU, 2009; VERRET et al., 2012).

Os dados oriundos do SSRS BR indicam que a maioria dos participantes alcançou um escore global das HS inferior a 35 na fase pré-intervenção. Algumas pesquisas têm demonstrado que pessoas com DI apresentam um repertório de HS baixo (MATSON et al., 2003; SANTOS, 2007; JAPUNDZA-MILISAVLJEVIC; DJURIC-ZDRAVKOVIC; MACESIC-PETROVIC, 2010; SMITH; MATSON, 2010). A literatura tem apontado que o baixo escore nas HS está associado a problemas psicossociais, tais como a depressão, ansiedade, estresse, isolamento social, agressividade, hiperatividade e baixa autoestima (BANDEIRA et al., 2005; DEL PRETTE; DEL PRETTE, 2005; MARTON et al., 2009). Entretanto não foram encontrados estudos que comprovem a associação dos problemas psicossociais em pessoas com DI, apesar de apontarem que este público está mais propenso a tais problemas (CHADWICK; TAYLOR; ABBA, 2005; EMERSON; EINFELD; STANCLIFFE, 2010; KLEEFMAN et al., 2014).

Rosni-Pinolla, Del Prette e Del Prette (2007) apontam que as pessoas com DI possuem um escore baixo nos fatores responsabilidade, autocontrole e cooperação com os pares, o que indica necessidade de maior estimulação nesses aspectos. No programa proposto por este estudo, uma combinação de fatores (jogos com invenção de regras; ginástica circense com expressão de sentimentos; diferentes cumprimentos nas lutas; autonomia em expressar-se) além da estrutura organizacional da aula, com momentos bem definidos sugere ter influenciado o contato visual entre os participantes, cumprimento das regras estabelecidas e colaboração com os colegas e professora (FERREIRA, 2014).

Algumas pesquisas têm apontado que as pessoas com DI tendem a evitar o contato visual durante a comunicação, possuem dificuldades em seguir as regras e constantemente interrompem as atividades em que estão inseridos (MATSON et al., 2003; SMITH; MATSON, 2010). Além disso, esse público pode encontrar dificuldades em estabelecer a comunicação devido a não estruturação das representações simbólicas (GONÇALVES et al., 2006). Essas descobertas apoiam a ideia de que as atividades em grupo com uso de situaçóes de enfrentamento e constante argumentação do professor, típicas das dinâmicas da Educação Física, podem levar à aquisição de comportamentos sociais desejáveis, favorecendo, sobretudo a comunicação.

Ao analisar as classes das HS isoladamente não foram encontradas mudanças positivas, porém destaca-se um aumento nas classes Autodefesa, Autocontrole, Cooperação e Asserção Positiva. Tais evidências podem indicar que as atividades empregadas no programa de Educação Física auxiliaram nessa melhora, uma vez que, os jogos cooperativos apresentam componentes como oferecer auxílio aos colegas, solicitar ajuda e compartilhar materiais. Nos esportes e nas lutas é comum situações de conflito, pressão, além de tomada de decisão em relação ao comportamento injusto, sendo comportamentos associados ao autocontrole e asserção positiva, respectivamente (DEL PRETTE; DEL PRETTE, 2011). Os conteúdos ginástica e dança favorecem as habilidades no relacionamento interpessoal, estimulando a empatia entre os participantes. 


\section{Conclusốes}

Com base nos resultados obtidos pelo SSRS-BR, foi possível verificar que, após o programa de Educação Física com base na cultura corporal de movimento, os participantes obtiveram um aumento nas Habilidades Sociais. A maioria dos participantes atingiu uma mudança positiva confiável no escore global das Habilidades Sociais.

Além disso, todas as classes de Habilidades Sociais avaliadas obtiveram um percentual de melhora e incidiram de forma estatisticamente significante no escore Global das Habilidades Sociais. Assim, o programa de Educação Física pode proporcionar ganhos para as crianças com DI, principalmente nas categorias responsabilidade, asserção positiva e autocontrole.

Como sugestão para estudos futuros, recomenda-se a avaliação das habilidades sociais com base em outras escalas construídas para essa finalidade. Ressalta-se a importância de analisar o efeito de programas baseados em outras abordagens de ensino da Educação Física, ou ainda junto a populaçóes com outros tipos de necessidades especiais. Assim, espera-se reforçar o papel da Educação Física na aquisição e aprimoramento de habilidades sociais junto a populaçôes com diferentes características e em contextos diversificados.

\section{REFERÊNCIAS}

ANDRADE, D.L.; HOLSBACH, M.L.C.G. As atividades lúdicas em terapia ocupacional. Revista Multitemas, Campo Grande, n.23, p.50-56, 2001.

BANDEIRA, M. et al. Comportamento assertivo e sua relação com ansiedade, locus de controle e auto-estima em estudantes universitários. Estudos de Psicologia, Campinas, v.22, n.2, p.111-121, 2005. BARBOSA, M.V.L.; DEL PRETTE, Z.A. Habilidades sociais em alunos com retardo mental: análise de necessidades e condiçôes. Revista Educação Especial, Santa Maria, v.20, p.31-53, 2002.

BIANCONI, E.; MUNSTER, M. Avaliação de aspectos psicomotores em jovens e adultos com deficiência intelectual antes e após um programa de educação física. In: CONGRESSO BASILEIRO MULTIDISCIPLINAR DE EDUCAÇÃO ESPECIAL, 7., 2011, Londrina. Anais eletrônicos... Londrina: UEL, 2011. Disponível em: <http://www.uel.br/eventos/congressomultidisciplinar/pages/ arquivos/anais >. Acesso em: 21 abr. 2014.

BLUECHARDT, M.H.; SHEPHARD, R.J. Using an extracurricular physical activity program to enhance social skills. Journal of Learning Disabilities, v.28, n.3, p.160-169, 1995.

BOLSONI-SILVA, A.T. Habilidades sociais: breve análise da teoria e da prática à luz da análise do comportamento. Interação em Psicologia, Curitiba, v.6, n.2, p.233-242, 2002.

CARDOZO, A.; SOARES, A.B. A influência das habilidades sociais no envolvimento de mães e pais com filhos com retardo mental. Aletheia, Canoas, n.31, p.39-53, 2010.

CASTELLANI FILHO, L. et al. Metodologia do ensino de Educação Física. 2.ed. São Paulo: Cortez, 2009.

CHADWICK, P.; TAYLOR, K.N.; ABBA, N. Mindfulness groups for people with psychosis.

Behavioural and Cognitive Psychotherapy, v.33, n.3, p.351-359, 2005.

CIDADE, R.E.; FREITAS, P.S. Educação física e inclusão: consideraçôes para a prática pedagógica na escola. Revista integração, Brasília, DF, v.14, p.27-30, 2002.

COOK, F; OLIVER, C. A review of defining and measuring sociability in children with intellectual disabilities. Research in developmental disabilities, v.32, n.1, p.11-24, 2011. 
DE AGUIAR, A.A.R. et al. Método JT na Educação Especial: resultados de um programa de habilidades sociais-comunicativas com deficientes mentais. Revista Educação Especial, Santa Maria, v.23, n.33, p.241-256, 2010.

DE FREITAS, A.B.M. Enunciação e autoria via comunicação alternativa e interlocução mediadora. Revista Brasileira de Lingüistica Aplicada, Belo Horizonte, v.12, n.1, p.165-180, 2012.

DE SÁ, L.G.C.; DEL PRETTE, Z.A.P. Dependentes químicos sob tratamento ambulatorial: análise de impacto sobre habilidades sociais. In: VILLA, M.; AGUIAR, A.; DEL PRETTE, Z.A (Org.). Intervençóes baseadas em evidências: aplicaçôes do Método JT. São Carlos: EDUFSCar, 2012. p.127-147.

DEL PRETTE, Z.A.P.; BARRETO, S.D.O.; FREITAS, L.C. Habilidades sociais na comorbidade entre dificuldades de aprendizagem e problemas de comportamento: uma avaliação multimodal. Revista Psico, Rio Grande do Sul, v.42, n.4, p.503-510, 2011.

DEL PRETTE, Z.A.P.; DEL PRETTE, A. Psicologia das habilidades sociais na infância: teoria e prática. 5.ed. Petrópolis: Vozes, 2011.

DEL PRETTE, Z.A.; DEL PRETTE, A. Psicologia das habilidades sociais: Diversidade teórica e suas implicaçóes. Petropólis: Vozes, 2009.

DEL PRETTE, Z.A.P.; DEL PRETTE, A. Significância clínica e mudança confiável na avaliação de intervençóes psicológicas. Psicologia: teoria e pesquisa, Brasília, DF, v.24, n.4, p.497-505, 2008.

DEL PRETTE, Z.A.P.; DEL PRETTE, A. Psicologia das habilidades sociais na infância: teoria e prática. Petrópolis: Vozes, 2005.

EMERSON, E.; EINFELD, S.; STANCLIFFE, R.J. The mental health of young children with intellectual disabilities or borderline intellectual functioning. Social psychiatry and psychiatric epidemiology, v.45, n.5, p.579-587, 2010.

FERREIRA, B.C.; DEL PRETTE, Z.A.P. Programa de expressividade facial de emocoes e habilidades sociais de criancas deficientes visuais e videntes. Psicologia: Reflexão e Crítica, Rio Grande do Sul, v.26, n.2, p.327-338, 2013.

FERREIRA, E.F. Habilidades sociais e deficiência intelectual: influência de um programa de educação física baseado na cultura corporal. 2014. 264f. Dissertação (Mestrado em Educação Especial) Departamento de Educação Especial, Universidade Federal de São Carlos, São Carlos, 2014.

FREITAS, L.C.; DEL PRETTE, Z.A.P. Comparando autoavaliação e avaliação de professores sobre as habilidades sociais de crianças com deficiência mental. Interpersona, Vitória, v.4, n.2, p.183-193, 2010a.

FREITAS, L.C.; DEL PRETTE, Z.A.P. Validade de critério do Sistema de Avaliação de Habilidades Sociais (SSRS-BR). Psicologia: Reflexão e Crítica, Rio Grande do Sul, v.23, n.3, p.430-439, 2010 b.

GIL, A. C. Métodos e técnicas de pesquisa social. 6.ed. São Paulo: Atlas, 2008.

GONÇALVES, E.S.; MURTA, S.G. Avaliaçáo dos efeitos de uma modalidade de treinamento de habilidades sociais para crianças. Psicologia: reflexão e crítica, Rio Grande do Sul, v.21, n.3, p.430-436, 2008. GONÇALVES, V.O. et al. Educação Física adaptada e avaliação: um caminho para o trabalho motor em alunos com deficiência mental. Pensar a Prática, Goiânia, v.7, n.2, p.231-244, 2006.

GORLA, J. I.; RODRIGUES, J. L.; PEREIRA, V. R. Avaliação e intervenção na educação física para portadores de deficiência mental. Arquivos do Museu Dinâmico Interdisciplinar, Maringá, v.6, n.1, p.15-19, 2002.

GOUDAS, M.; MAGOTSIOU, E. The effects of a cooperative physical education program on students' social skills. Journal of applied sport Psychology, v.21, n.3, p.356-364, 2009.

GRESHAM, F.M.; VAN, M.B.; COOK, C.R. Social skills training for teaching replacement behaviors: Remediating acquisition deficits in at-risk students. Behavioral Disorders, v.3, p.363-377, 2006. 
JACOBSON, N.S.; TRUAX, P. Clinical significance: a statistical approach to defining meaningful change in psychotherapy research. Journal of consulting and clinical psychology, v.59, n.1, p.12, 1991. JAPUNDZA-MILISAVLJEVIC, M.; DJURIC-ZDRAVKOVIC, A.; MACESIC-PETROVIC, D. The socially acceptable behavioural patterns in children with intellectual disabilities. Procedia-Social and Behavioral Sciences, v.5, p.37-40, 2010.

KLEEFMAN, M. et al. The effectiveness of Stepping Stones Triple P parenting support in parents of children with borderline to mild intellectual disability and psychosocial problems: a randomized controlled trial. BMC medicine, v.12, n.191, p.1-10, 2014.

LABAN, R. Dança educativa moderna. São Paulo: Ícone, 1990.

MARTON, I. et al. Empathy and social perspective taking in children with attention-deficit/ hyperactivity disorder. Journal of abnormal child psychology, v.37, n.1, p.107-118, 2009.

MATSON, J.L. et al. A comparison of social and adaptive functioning in persons with psychosis, autism, and severe or profound mental retardation. Journal of Developmental and Physical Disabilities, v.15, n.1, p. 57-65, 2003.

MAUERBERG-DE CASTRO, E. et al. Educação física adaptada inclusiva: impacto na aptidão física de pessoas com deficiência intelectual. Revista Ciência em Extensão, São Paulo, v.9, n.1, p.35-61, 2013.

MAUERBERG-deCASTRO, E. Atividade fisica adaptada. Ribeirão Preto: Tecmedd, 2011. PARK, K. L.; LOMAN, S.; MILLER, M.A. Social skills. In: OAKLAND, T.; HARRISON, P.L. (Org.). Adaptive Behavior Assessment System-II: Clinical Use and Interpretation. Londres: Academic Press, 2008. p.196-217.

PÉREZ-RAMOS, A.M. et al. A importância da avaliação da competência social em educandos com deficiência intelectual. Psic: revista da Vetor Editora, São Paulo, v.4, n.1, p.30-41, 2003.

ROSIN-PINOLA, A.R.; DEL PRETTE, Z.A.P.; DEL PRETTE, A. Habilidades sociais e problemas de comportamento de alunos com deficiência mental, alto e baixo desempenho acadêmico. Revista Brasileira de Educação Especial, Marília, v.13, n.2, p.239-256, 2007.

SANTOS, A.S.P.G.D. Estudo psicométrico da escala de comportamento adaptativo versão portuguesa (Ecap). 2007. 325f. Tese (Doutorado em Educação Física) - Faculdade de Motricidade Humana, Universidade Técnica de Lisboa, Portugal, 2007.

SCHALOCK, R.L. et al. Intellectual disability: Definition, classification, and systems of supports. Washington: ERIC, 2010.

SMITH, K.R.; MATSON, J.L. Social skills: Differences among adults with intellectual disabilities, co-morbid autism spectrum disorders and epilepsy. Research in Developmental Disabilities, v.31, n.6, p.1366-1372, 2010.

VAN NIEUWENHUIJZEN, M.; VRIENS, A. (Social) Cognitive skills and social information processing in children with mild to borderline intellectual disabilities. Research in developmental disabilities, v.33, n.2, p.426-434, 2012.

VERRET, C. et al. A physical activity program improves behaviour and cognitive functions in children with ADHD: An exploratory study. Journal of Attention Disorders, v.16, n.1, p.71-80, 2012.

VILLA, M.; AGUIAR, A.; DEL PRETTE, Z. Intervençôes baseadas em evidências: aplicações do método JT. São Carlos: EDUFSCar, 2012.

Recebido em: 05/06/2016

Reformulado em: 30/01/2017

Aprovado em: 02/02/2017 